Apuntes Universitarios, 2020: 10(3), julio-setiembre

ISSN: 2304-0335 DOI: https://doi.org/10.17162/au.v10i3.454

\title{
Percepción de la población respecto al ruido producido por el transporte público en el distrito de Barranca, Lima, Perú
}

\author{
Perception from population about noise produced by public transport in district of \\ Barranca, Lima, Peru
}

\author{
Miguel Ángel Castillo Corzo ${ }^{1 a}$, Jorge Moisés Minaya Martínez ${ }^{2}$ y \\ Adriana María Castillo Corzo ${ }^{3}$ \\ Universidad Nacional Mayor de San Marcos, Perú ${ }^{1}$ \\ Universidad César Vallejo, Perú ${ }^{2}$ \\ Universidad Nacional José Faustino Sánchez Carrión, Perú ${ }^{3}$
}

(iD) Orcid ID: http//orcid.org/0000-0002-2652-799X ${ }^{1}$

(iD) Orcid ID: http://orcid.org/0000-0003-2964-3387²

(iD) Orcid ID: http://orcid.org/0000-0003-0786-60293

Recibido: 09 de mayo 2019

Aceptado: 15 de enero 2020

\begin{abstract}
Resumen
En el presente trabajo se muestran los resultados de la investigación realizada sobre la percepción de los habitantes del distrito de Barranca en Lima, Perú, respecto a la influencia del ruido ambiental producido por el transporte vehicular. Como metodología de recolección de información se aplicó una encuesta, con la que se confirmó si las personas consideraban que el ruido vehicular afectaba su quehacer diario y luego, a los que respondieron positivamente, se les aplicó el instrumento con cinco preguntas y cuatro niveles de respuesta. Los resultados se trataron con estadística descriptiva (análisis de frecuencias) e inferencial (varianza de Friedman) a fin de establecer el grado de relevancia de cada factor. Se obtuvo que la mayoría considera que el ruido vehicular no afecta $(58.2 \%)$ y los que consideran que sí afecta ubican a la falta de concentración como el problema más importante, seguido del estrés y la agresividad. Se corrobora que existe diferencia significativa entre los resultados de las preguntas con nivel de confianza de 95\%; asimismo, las preguntas se ubicaron en tres grupos de acuerdo a la prueba de Friedman (mal humor y pérdida de audición, estrés y agresividad, concentración), los cuales presentan igualdad estadística intragrupos y diferencias extragrupos.
\end{abstract}

Palabras clave: Contaminación sonora, percepción, población, ruido, tráfico vehicular, transporte

${ }^{\mathrm{a} C}$ Correspondencia al autor

E-mail: migang2000@gmail.com 


\begin{abstract}
The present study shows the results of the research carried out on the perception of the inhabitants of the Barranca district in Lima, Peru, regarding the influence of environmental noise produced by vehicular transport. As a methodology to collect information, a survey was applied, with which it was confirmed if people considered that vehicular noise affected their daily work and then, to those who responded positively, the instrument was applied with five questions and four levels of response. The results were treated with descriptive statistics (frequency analysis) and inferential statistics (Friedman's Variance) to establish the degree of relevance of each factor. It was obtained that the majority considers that vehicle noise does not affect $(58.2 \%)$ and that those who consider that it does affect place lack of concentration as the most important problem, followed by stress and aggressiveness. There is a significant difference between the results of the questions with a 95\% confidence level. Likewise, the questions were placed in three groups according to the Friedman test (bad mood and hearing loss, stress and aggressiveness, concentration), which present statistical equality within groups and differences outside groups.
\end{abstract}

Keywords: Sound pollution, perception, population, noise, vehicular traffic, public transportation

\title{
Introducción
}

El ruido es considerado un agente contaminante que puede llegar a producir efectos fisiológicos (p. ej. pérdida de audición) y psicológicos (irritabilidad) nocivos para las personas (Nriagu, 2019). La causa principal del ruido es la actividad humana, principalmente por el transporte, las labores de construcción civil, la industria, entre otras. El ruido se mide en decibelios (dB), para lo cual se utilizan equipos denominados sonómetros. Según un informe de la Organización Mundial de la Salud (OMS), se considera los 50 dB como el límite superior deseable (Zamorano et al., 2019).

Uno de los problemas que afectan la salud de las personas es la que se genera por la sobreexposición a los ruidos generados en nuestro medio ambiente; a esto se le denomina "contaminación sonora," y puede producir afecciones fisiológicas y psicológicas. De acuerdo con Auger, et al. (2018) una de las repercusiones más comunes es la hipertensión, la cual se produce porque el ruido provoca la secreción de adrenalina, noradrenalina y cortisolhormonas endocrinas que causan vasoconstricción y tienen un impacto cardiovascular. Cuando se refiere al ruido como sinónimo de contaminación acústica, se está haciendo referencia a un sonido con intensidad alta que puede llegar a afectar negativamente la salud humana (Maijala et al., 2018). Los niveles de ruido permitidos dependen de las legislaciones propias de cada país; sin embargo, la OMS ha establecido valores límites y ha generado informes que confirman la relación del ruido con problemas auditivos y afecciones de salud tanto mentales cono fisiológicas (Amable et al., 2017). 
A nivel mundial se han realizado investigaciones sobre ruido ambiental $\mathrm{y}$, en específico, sobre el efecto del ruido vehicular como principal factor de anomalías, en los que se estudió la percepción de las personas respecto al efecto del ruido (Kestera, Zarazua de Rubens, Sovacool y Noel, 2019; Liang, Chen y Li, 2020). Igualmente, en el ámbito del Perú se encuentran algunos estudios relacionados con la medición de la percepción del efecto del ruido, como el trabajo de Moya (2017) llevado a cabo en el distrito Miraflores de Lima. A la par, Loayza y Rodrígue z (2017) investigaron sobre los niveles de ruido y la percepción de este por parte de los habitantes del asentamiento humano 200 Millas en el Callao, Perú. En todos los casos se demuestra que la percepción de las personas es un instrumento de suma importancia a ser adicionado a las mediciones técnicas de ruido, a fin de efectivizar la caracterización y manejo de este problema ambiental (Ba y Kang, 2019).

Por otro lado, cabe resaltar que el marco jurídico peruano posee ordenanzas, decretos, leyes y resoluciones en materia de contaminación ambiental y sonora en los que se establece el límite permisible medido en decibeles $\mathrm{dB}$; y las organizaciones gubernamentales locales son las encargadas de hacer cumplir estas normativas. Entre estas se puede citar el Decreto Supremo No 085-2003-PCM y la Resolución Ministerial N No227-2013-MINAM.

En el caso específico del área de estudio, gran parte de la ciudad del distrito de Barranca, está sujeta a problemas por ruido vehicular, ya que por su territorio atraviesa la Carretera Panamericana Norte y siendo capital de la provincia, el tráfico vehicular urbano es de importancia debido a los niveles de ruido que el mismo provoca. Por lo anterior, se desarrolló la presente investigación, el cual—a través de encuestas a una muestra representativa de la población-se logró establecer el nivel de percepción de los habitantes respecto a los

principales problemas generados por el ruido vehicular, aplicando estadística descriptiva e inferencial para medir de esta manera el grado de significancia de las respuestas obtenidas. Esto puede servir de base para una campaña de educación y concientización a la población, con lo que se puede aportar a la solución y/o mitigación del problema.

\section{Materiales y métodos}

La población de estudio estuvo conformada por los habitantes del distrito de Barranca de la región Lima, que consta de aproximadamente 80000 habitantes según el último censo poblacional (INEI, 2018). La muestra fue calculada aproximando a una población infinita (Manzano, 1996). Se aplicó la ecuación descrita por Morales (2012) para el cálculo de muestras en poblaciones infinitas: 


$$
n=\frac{\left(Z^{2} * p * q\right)}{e^{2}}
$$

Dónde: $Z$ es el cuartil de la distribución normal estándar correspondiente a $\alpha$ (nivel de confianza); $p$ es porcentaje estimado de la población, $q=100-p$; $e$ margen de error aceptable y $n$ es el número de muestras a tomar.

Con nivel de confianza de $95 \%(\alpha=0.5), Z$ es de 1.96 y error permisible de $5 \%$, se obtuvo 246 individuos. Para obtener mayor margen de confianza se adicionaron 10 individuos a la muestra, la cual quedó constituida por 256 individuos. Los datos fueron recolectados en el año 2017 a una muestra aleatoria de pobladores del distrito de Barranca en Lima y tabulados en hojas de cálculo de Microsoft Excel, para luego ser tratados estadísticamente.

Como técnica de recolección de información se aplicó una encuesta, la que consistió en cinco preguntas condicionadas a si los encuestados consideraban que eran afectados o no y con cuatro niveles de respuestas en el caso afirmativo (según el formato mostrado en la Tabla 1), lo cual es una adaptación del cuestionario propuesto y validado por Purdy y Williams (2002). De este instrumento se tomaron los ítems correspondientes al ruido vehicular y que presentó una validez, medida a través del coeficiente Alfa de Cronbach de 0.81, considerado bueno. Asimismo, se tomaron en cuenta las propuestas de González (2006) y Rodríguez y Baldeón (2018).

Tabla 1. Formato de encuesta aplicada a la muestra de estudio

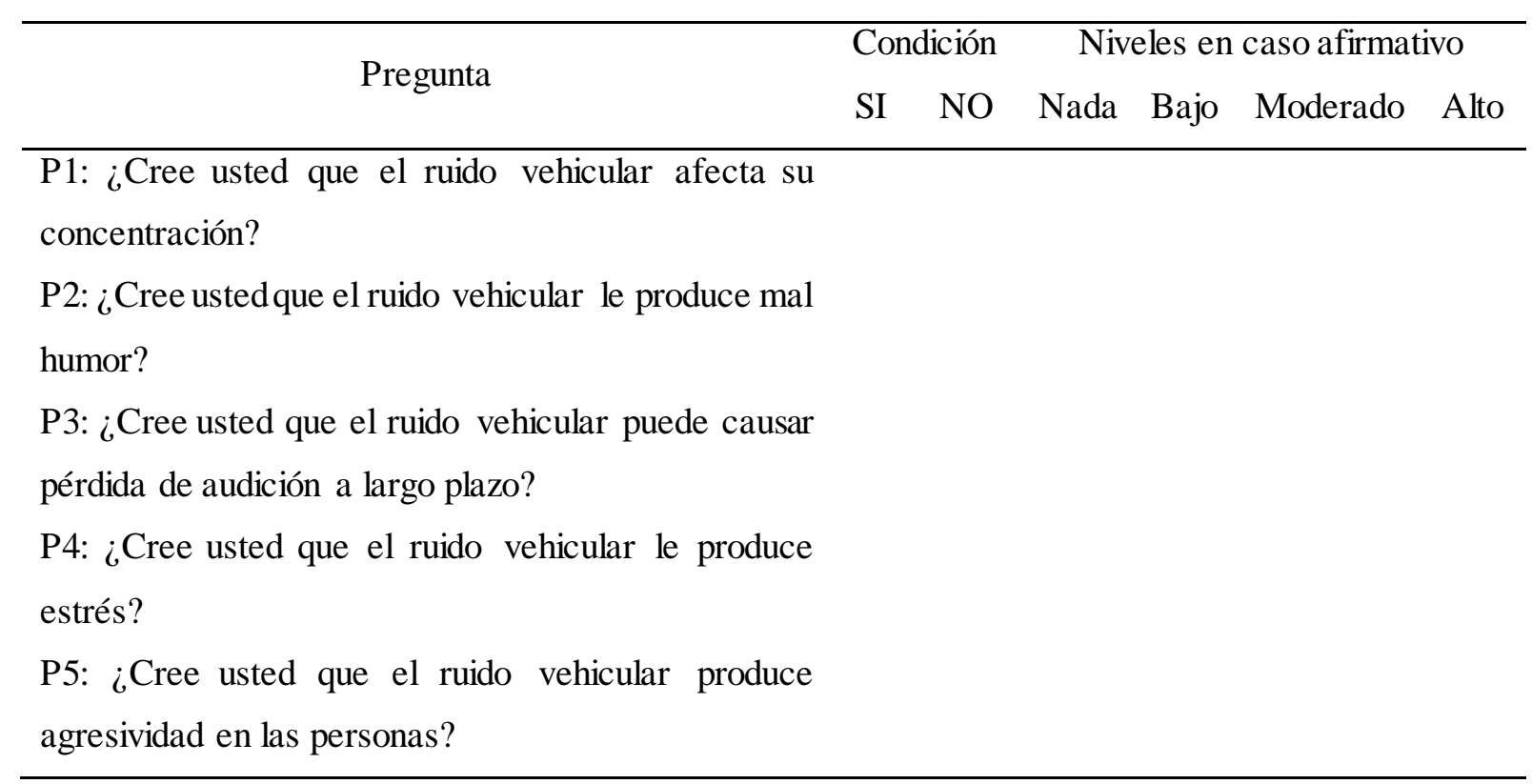

Fuente: Elaboración propia. 


\section{Análisis estadístico}

A los resultados obtenidos se les aplicó estadística descriptiva, con el fin de analizar el nivel de percepción de los pobladores respecto a los niveles de ruido ambiental producto del transporte. También se aplicó la prueba de Análisis de varianza no paramétrica de Friedman, para establecer el nivel de asociación entre las respuestas a las preguntas planteadas. El anális is estadístico se realizó mediante el software InfoStat versión 2018.

\section{Resultados}

Las respuestas de los encuestados respecto a si el ruido vehicular afectaba o no a las personas, fueron graficadas y se muestran en la figura 1.

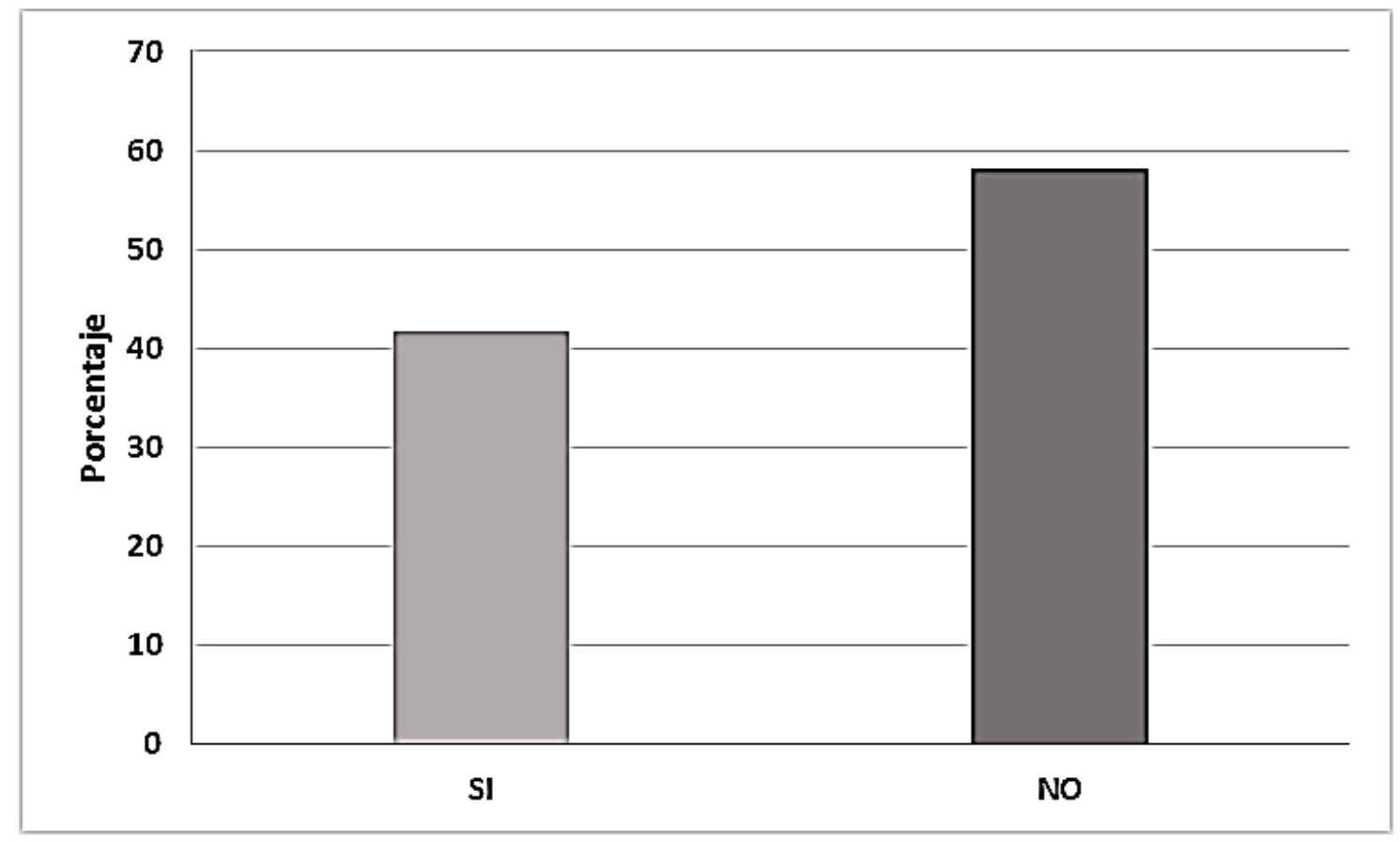

Figura 1. Distribución de la respuesta de los encuestados respecto al efecto del ruido vehicular sobre las personas. Fuente: Elaboración propia.

Se observa que, a pesar del malestar que causa el ruido del transporte en la zona de estudio, la mayoría de los encuestados $(58.2 \%)$ manifestó que el nivel de ruido no era suficiente para causar daño a las personas.

Con los datos obtenidos se realizó el gráfico mostrado en la figura 2, respecto a la percepción de los encuestados sobre la afectación del ruido producido por el transporte. 


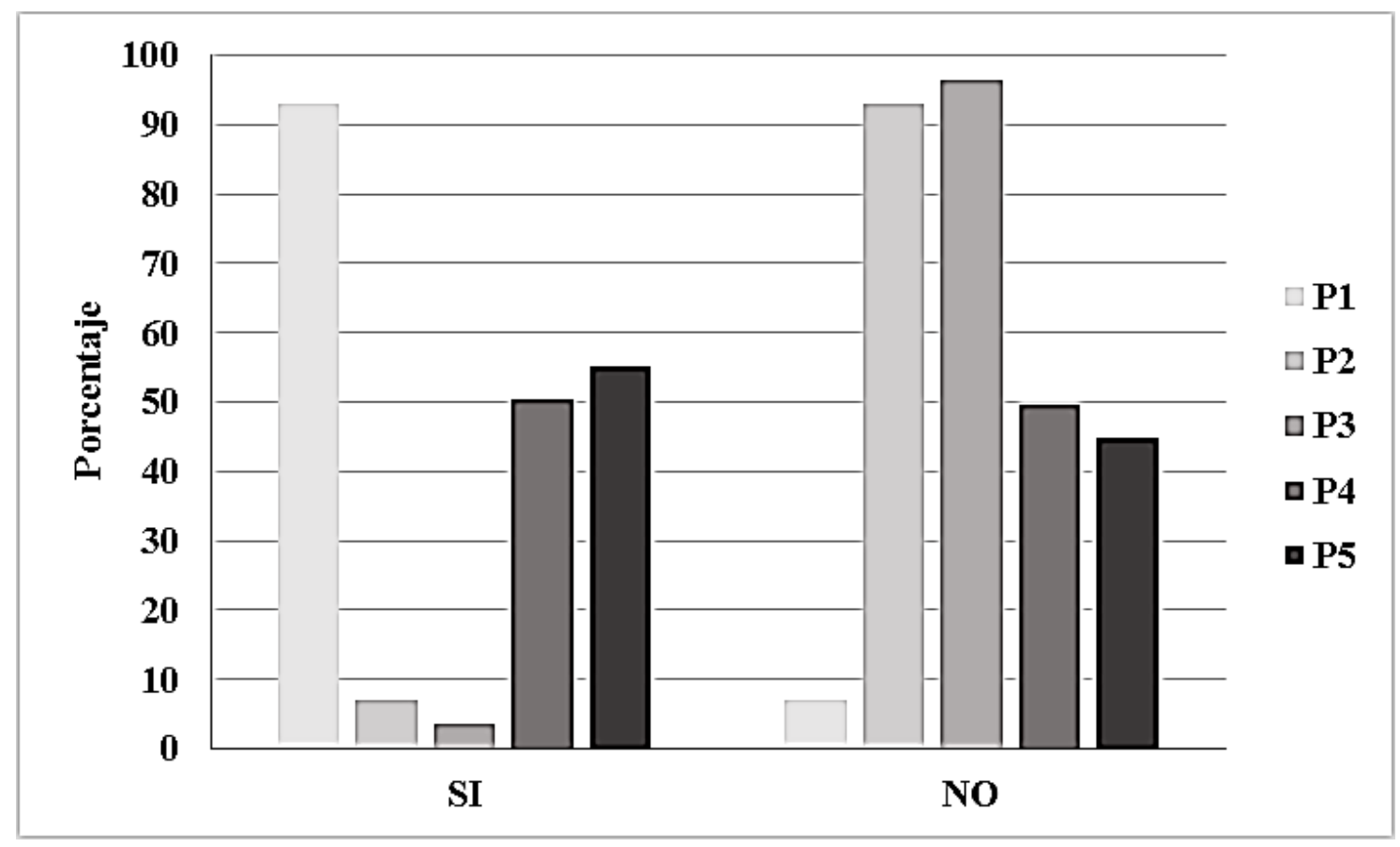

Figura 2. Percepción del efecto del ruido por transporte en la población estudiada. Fuente: Elaboración propia.

Se observa que, para el 93\% de los encuestados, el ruido producido por el transporte afecta la concentración de las personas; asimismo, una proporción importante considera que este influye en el nivel de estrés y de agresividad (50.4 y 55.1\%). En contraste, la mayoría considera que no hay influencia del ruido sobre el mal humor y la pérdida de audición a largo plazo (93 y 96.5\%). Igualmente, una cantidad importante también considera que no influye en el estrés y la agresividad (49.6 y 44.9\%).

En la figura 3 se muestran gráficamente los resultados de la percepción en cuanto a los niveles de afectación detectados en cada una de las preguntas. 


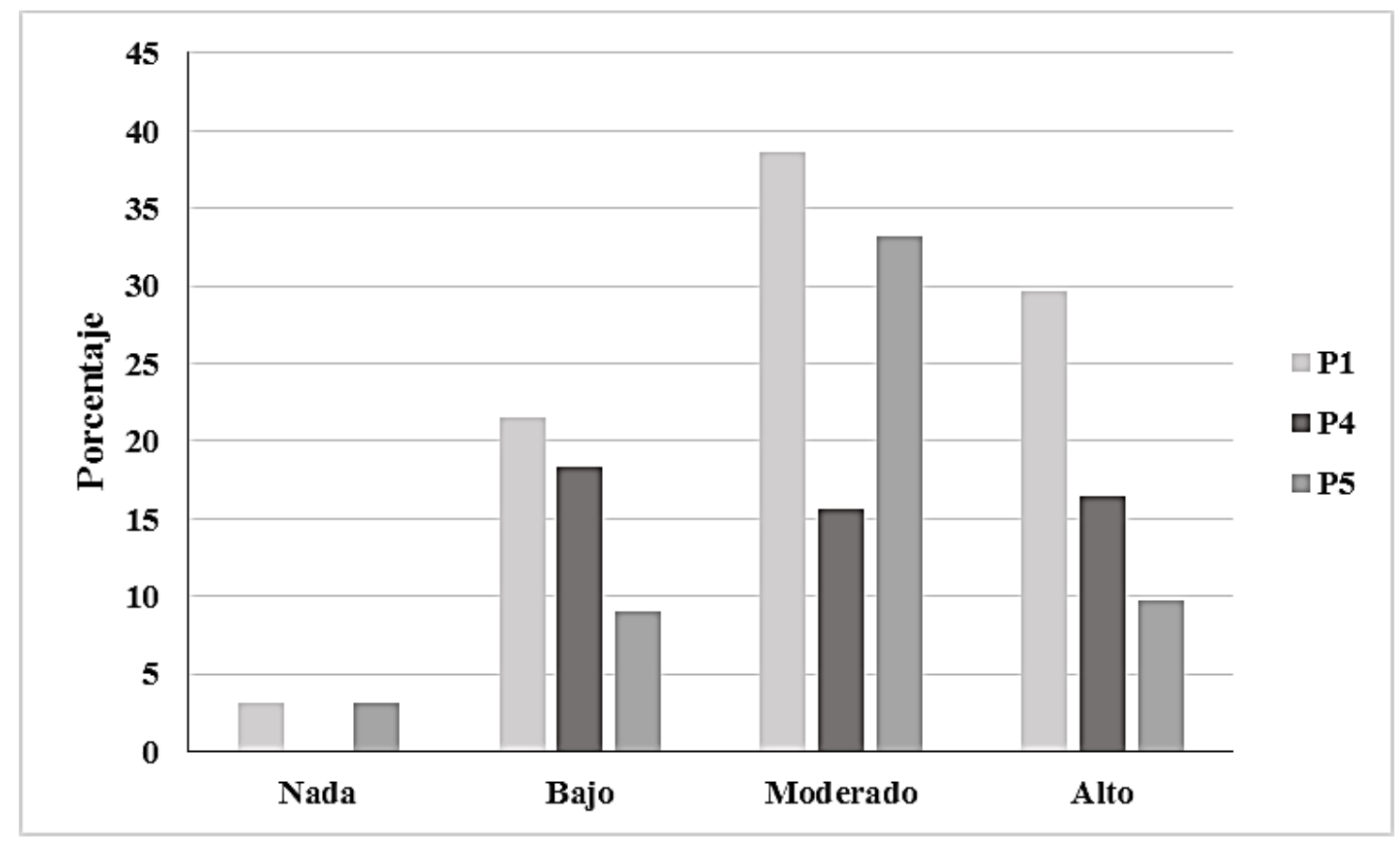

Figura 3. Distribución porcentual de las opiniones respeto a la afectación del ruido sobre la concentración, el estrés y la agresividad. Fuente: Elaboración propia.

Se observa que la falta de concentración es afectada de un nivel moderado a alto (68.4\%) con predominancia de la percepción de moderada con 38.7\%. En el caso del estrés, se observan porcentajes más constantes entre los niveles, con porcentajes de 18.4\% (bajo), 15.6\% (moderado) y $16.4 \%$ (alto). Para la mayoría de los encuestados, el ruido vehicular incide sobre la agresividad de las personas de forma moderada (33.2\%) y porcentajes similares consideran que el efecto es bajo o alto $(9.0$ y $9.8 \%)$ y una menor cantidad se inclinó por opinar que no afecta en nada $(3.1 \%)$.

Un análisis de correlación de Pearson entre los dos factores más relevantes (concentración y estrés) arrojó como resultado un $\mathrm{R}$ de 0.82 , el cual representa una asociación positiva muy fuerte (Hopkins, 2016). Lo anterior sugiere que la pérdida de concentración debida al ruido, influye también en el nivel de estrés.

En la tabla 2 se muestra un resumen del conteo de respuestas de las personas que respondieron de manera afirmativa respecto a la influencia del ruido vehicular sobe el comportamiento de las personas en el área de estudio. 
Tabla 2. Resumen del conteo de niveles de afectación en las personas que respondieron de manera afirmativa.

\begin{tabular}{cccccc}
\cline { 2 - 4 } & \multicolumn{5}{c}{ Nivel } \\
\cline { 1 - 3 } Pregunta & Alto & Bajo & Moderado & Nada & Total \\
\hline P1 & 76 & 55 & 99 & 8 & 238 \\
P2 & 9 & 0 & 9 & 0 & 18 \\
P3 & 0 & 0 & 9 & 0 & 9 \\
P4 & 42 & 47 & 40 & 0 & 129 \\
P5 & 25 & 23 & 85 & 8 & 141 \\
Total & 152 & 125 & 242 & 16 & 535 \\
\hline
\end{tabular}

Fuente: Elaboración propia.

A partir de la tabla 2, se realizó un análisis comparativo a través de una prueba de varianza no paramétrica de Friedman (tabla 3), la que demostró que existen diferencias significativas entre las respuestas de las personas sobre la afectación del ruido vehicular (pvalor $<0.05)$.

Tabla 3. Resultados de la prueba de varianza no paramétrica de Friedman

\begin{tabular}{ccccccc}
\hline Tratamiento & Suma (Ranks) & Media (Ranks) & $\mathrm{n}$ & \multicolumn{3}{c}{ Ubicación } \\
\hline P3 & 6.00 & 1.50 & 4 & $\mathrm{~A}$ & & \\
P2 & 7.00 & 1.75 & 4 & $\mathrm{~A}$ & & \\
P4 & 13.00 & 3.25 & 4 & & B & \\
P5 & 14.50 & 3.63 & 4 & & B & \\
P1 & 19.50 & 4.88 & 4 & & & C \\
\hline
\end{tabular}

Medias con una letra común no son significativamente diferentes $(p>0.05)$

Fuente: Elaboración propia.

Se observa que el nivel de afectación considerado de mayor importancia fue el moderado, seguido por el alto. Muy pocas personas consideraron que no existía afectación. Igualmente se nota que P1 superó a las demás preguntas, es decir, fue la de mayor importancia. Se observa también que las respuestas a las preguntas P2 y P3 son estadísticamente iguales, lo que significa que, para los encuestados, la influencia del ruido vehicular sobre el mal humor y la pérdida de audición es equivalente. Finalmente, las respuestas a las preguntas P4 y P5 no presentan diferencias significativas; el efecto del ruido vehicular sobre la concentración mostró diferencia significativa respecto al resto, siendo este el factor de mayor relevancia para los encuestados. 


\section{Discusión}

El ruido vehicular es un problema ambiental que afecta a todas las concentraciones urbanas en el planeta; por tal motivo, la percepción de la población respecto al mismo es de suma importancia para generar políticas y estrategias mitigadoras. En el distrito de Barranca de Lima, Perú, se han reportado altos niveles de ruido, como lo precisa Castillo (2017), quien obtuvo medidas de ruido desde $81.6 \mathrm{~dB}$ a $84.9 \mathrm{~dB}$, valores que sobrepasan los límites permisibles establecidos en el Decreto Supremo Nº 085-2003-PCM (máximo 80 dB para zonas industriales). Sin embargo, los resultados mostraron que la mayoría de los encuestados (58.2\%) considera que el ruido vehicular no tiene algún efecto sobre las personas; esto no coincide con la investigación de Santos (2007) en la que se informó un porcentaje mayor de personas que consideraron que el ruido vehicular sí afecta la salud. Por su parte, Ramírez y Domínguez (2011) mencionan que el ruido vehicular incide negativamente en la calidad de vida de las personas, referenciando investigaciones a nivel mundial. Solís (2013) concluyó que el ruido debido al tráfico vehicular en Cercado de Lima fue considerado por los encuestados como el de mayor relevancia, mostrando una percepción negativa generalizada; esto no coincide con los resultados obtenidos en la presente investigación. De igual manera, Perea y Marín (2014) obtuvieron que, al encuestar a habitantes de la ciudad de Cali en Colombia, el 77\% consideró que en que la zona se presenta niveles altos de ruido, que no es coincidente con lo obtenido en este estudio.

Todo lo anterior demuestra que un porcentaje importante considera el ruido como un problema relevante, como lo demostró Peña (2013) con mediciones realizadas en el distrito de Barranca, las cuales superaron los límites legales establecidos para Perú. En la mayoría de las investigaciones revisadas las mediciones se hicieron en zonas metropolitanas con mayor afluencia de vehículos y mayor cantidad de habitantes, lo que pudo influir en la percepción. Asimismo, Lu et al. (2019) concluyen que los niveles de ruido y la percepción de las personas en zonas urbanas no depende solo de la cantidad de vehículos, sino de las condiciones de las vías y la presencia o no de semáforos.

Según la percepción de las personas, el nivel de afectación del ruido vehicular en el distrito de Barranca se puede considerar de moderado a alto, lo que es coincidente con lo reportado en la ciudad de Tacna por Yagua (2016), quien concluyó que para la mayoría el ruido debido al transporte es el más relevante. Los niveles de ruido medidos en la ciudad de Piura, en Perú, también excedieron los valores legales permitidos, demostrándose que hay un problema común asociado a la presencia de los vehículos y que la percepción de las personas está acorde 
con los mismos (Timaná, 2017). Otra condición o factor que puede influir en la percepción de la afectación del ruido en determinada comunidad es la presencia de vegetación, como lo manifestaron Ba y Kang (2019), al estudiar la percepción del ruido relacionada con la presencia de plantas aromáticas y observaron que cuanto mayor era la intensidad de la fragancia, más cómodas y menos molestas estaban las personas, respecto al ruido.

En opinión de Solís (2013), el estrés constituye la mayor consecuencia del ruido vehicular en el Cercado de Lima, seguido por la agresividad y la falta de concentración. La diferencia de criterios y de percepción se debe a la temporalidad de las mediciones y la percepción subjetiva propia de los encuestados por haberse realizado en dos lugares diferentes. Por su parte, Baca y Seminario (2012), reportaron resultados que coinciden con lo obtenido en la investigación; sin embargo, se debe recalcar que el estudio citado se realizó en un campus universitario, lo que evidentemente produce un sesgo hacia la concentración como factor importante. Por otro lado, Chávez (2009) menciona que, a pesar de que los problemas auditivos son considerados como los de mayor importancia respecto al efecto del ruido, su resultado sobre la falta de concentración se muestra también como un problema relevante en la actualidad, como conclusión similar en Quevedo y Revolledo (2015) y Vásquez (2017).

El estrés y la agresividad en las personas también son consecuencias importantes del ruido vehicular en la zona de estudio, lo que coincide con Ramírez, Domínguez y Barrero (2011), quienes consideraron que los altos niveles de ruido vehicular son un problema de salubridad pública, causando daños sobre la población expuesta e incidiendo en otros problemas fisiológicos y psicológicos (como el estrés, principalmente). Asimismo, Saavedra (2011) estudió la percepción del ruido en las personas del distrito de Miraflores en Lima, obteniendo que los factores concentración, estrés y agresividad fueron los de mayor relevancia. Todo lo anterior sugiere que, independientemente del lugar que se tome como muestra, las percepciones de la población en cuanto a los efectos del ruido vehicular son similares, como lo evidencian la pérdida de concentración, el estrés y el aumento de la agresividad. Además de los problemas auditivos propios de la exposición a altos niveles de ruido ambiental, se han realizado investigaciones que relacionan este tipo de contaminación con otros problemas de salud, como el realizado por Halperin (2014) quien encontró relación entre el ruido y las perturbaciones del sueño, concluyendo que el ruido ambiental nocturno es la forma más preocupante de contaminación sonora por sus consecuencias para la salud, ya que la perturbación del sueño influye de manera directa o indirecta como mediadora en los sistemas biológicos. 
Con relación a la influencia del ruido en la salud de las personas, Yulianto y Saadah (2018) reportaron que el ruido ambiental influye en la calidad de vida de las personas, sobre todo cuando se está expuesto a altos niveles en el lugar de trabajo. Asimismo, Gupta y Ghatak (2011) observaron que las personas que estaban expuestas a altos niveles de ruido por tráfico sufrían, además de discapacidad auditiva, dolor de cabeza, ansiedad, hipertensión arterial, enfermedades cardiovasculares, irritabilidad e insomnio. Van Renterghem (2018) también menciona los efectos nocivos del ruido sobre la salud y propone la vegetación, como medida de reducción de estos, e indica que las características naturales visibles de buena calidad conducen a la restauración de la atención sostenida y al alivio del estrés, lo que contrarresta los efectos negativos de la exposición al ruido ambiental. Lo anterior indica que el ruido no solo afecta el estado de ánimo de las personas, sino también su salud física, por lo que se hace necesario estudiar más a profundidad este factor en el área de estudio, con la finalidad de mejorar la calidad de vida de los habitantes.

En otro orden, el análisis estadístico dio como resultado que existe diferencia significativa entre las respuestas dadas por los encuestados a las preguntas realizadas, por lo que se puede afirmar que existen diferentes percepciones subjetivas de los encuestados dependiendo de la pregunta que se les realizó. Resultados similares fueron reportados por Timaná (2017) en un estudio realizado en la ciudad de Piura, Perú. Igualmente, Salas y Barbosa (2016), al medir niveles de ruido ambiental en el Campus de la Universidad Nacional Toribio Rodríguez de Mendoza de Amazonas, Perú, obtuvieron diferencias estadísticamente significativas. Otro investigador que coincide con el estudio realizado fue Morales (2009), quien analizó el ruido vehicular en la ciudad de Madrid, España.

Finalmente, el uso del análisis de varianza de Friedman para relacionar parámetros o percepciones respecto al ruido vehicular ha sido poco reportado; sin embargo, se evidencia su uso en trabajos como el de Ramírez (2012) aplicado al ruido vehicular de la ciudad de Bogotá, en el que se demuestra la aplicabilidad del análisis, y que es coincidente con lo observado en la presente investigación. Es claro que el uso de la estadística no paramétrica no se arraiga en los investigadores; no obstante, la misma ha demostrado ser eficaz y en el caso particular, se observa que la misma refleja de manera significativa el comportamiento observado en las respuestas de los individuos. 


\section{Conclusiones}

De los resultados obtenidos, se concluye que los habitantes tienen percepciones diferentes respecto al nivel de afectación del ruido vehicular a las personas. Según las encuestas realizadas, las personas consideran la falta de concentración producida por efecto del ruido producido por el tráfico vehicular como la más relevante, aunque se observó también una importancia significativa del efecto del ruido sobre los niveles de estrés y en el aumento de la agresividad de las personas.

Asimismo, las respuestas a las preguntas formuladas presentaron diferencias significativas con base en el resultado de la prueba de varianza no paramétrica de Friedman, a partir de la cual se concluyó que para los encuestados el ruido ejerce efectos similares sobre el mal humor y la pérdida de audición y que, además, estos son los aspectos de menor relevancia. Además, el efecto sobre el estrés y la agresividad también fueron considerados similares y de relevancia intermedia, quedando el efecto del ruido vehicular sobre la falta de concentración como la consecuencia de mayor relevancia y estadísticamente diferente a los demás efectos.

\section{Referencias}

Amable, I., Méndez, J., Delgado, L., Acebo, F., De Armas, J., y Rivero, M.L. (2017). Contaminación ambiental por ruido. Revista Médica Electrónica, 39(3), 640-649.

Auger, N., Duplaix, M., Bilodeau-Bertrand, M., Lo, E. y Smargiassi, A. (2018). Environmenta 1 noise pollution and risk of preeclampsia. Environmental Pollution, 239, 599-606.

Ba, M., y Kang, J. (2019). Effect of a fragrant tree on the perception of traffic noise. Building and Environment, 156, 147-155.

Baca, W., y Seminario, S. (2012). Evaluación de impacto sonoro en la pontificia universidad católica del Perú (Tesis de pregrado). Pontificia Universidad Católica del Perú, Lima, Perú.

Castillo, M.A. (2017). La contaminación sonora en el distrito de barranca (Tesis de maestría). Universidad Nacional José Faustino Sánchez Carrión, Huacho, Perú.

Chávez, J.R. (2006). Ruido: Efectos Sobre la Salud y Criterio de su Evaluación al Interior de Recintos. Revista Ciencia \& Trabajo, 8(20), 42-46. 
González, S.A. (2006). Elaboración de una encuesta sobre percepción de ruido ambiental para ser aplicadas en familias del programa puente de la comuna de Chimbarongo (Tesis de licenciatura). Universidad Austral de Chile, Valdivia, Chile.

Gupta, S., y Ghatak, C. (2011). Environmental noise assessment and its effect on human health in an urban area. International Journal of Environmental Sciences, 1(7), 1954-1964.

Halperin, D. (2014). Environmental noise and sleep disturbances: A threat to health? Sleep Science, 7, 209-212.

Hopkins, W. G. (2016). A New View of Statistics. Disponible en https://www.sportsci.org/resource/stats/

Instituto Nacional de Estadística e Informática (2018). Perú: Perfil sociodemográfico informe nacional. Lima: INEI.

Kestera, J., Zarazua de Rubens, G., Sovacool, B. K. y Noel, L. (2019). Public perceptions of electric vehicles and vehicle-to-grid (V2G): Insights from a nordic focus group study. Transportation Research Part D, 74, 277-293.

Liang, L., Chen, S. y Li, P. (2020). The evaluation of vehicle interior impact noise inducing by speed bumps based on multi-features combination and support vector machine. Applied Acoustics, 163, 107212.

Loayza, R.M. y Rodríguez, M.M. (2017). Evaluación del ruido ambiental por tránsito aéreo y la percepción del impacto en la salud de los habitantes residentes del Asentamiento Humano 200 millas - Callao (Tesis de licenciatura). Universidad Nacional Mayor de San Marcos, Lima, Perú.

Maijala, P., Shuyang, Z., Heittola, T. y Virtanen, T. (2018). Environmental noise monitoring using source classification in sensors. Applied Acoustics, 129, 258-267.

Manzano, V. (1996). Acerca de la finitud en las poblaciones. Revista Estadística Española, $38(141), 237-244$.

Morales, J. (2009). Estudio de la influencia de determinadas variables en el ruido urbano producido por el tráfico de vehículos (Tesis doctoral). Universidad Politécnica de Madrid, España. 
Morales, P. (2012). Estadística aplicada a las ciencias sociales. Madrid: Universidad Pontificia Comillas.

Moya, T. (2017). Estimación de la contaminación acústica por ruido ambiental en la zona $8 \mathrm{c}$ del distrito de Miraflores - Lima (Tesis de licenciatura). Universidad Nacional Federico Villarreal, Lima, Perú.

Nriagu, J. (2019). Encyclopedia of Environmental Health. Environmental Noise. Michigan, EEUU: Elsevier.

Lu, X., Kang, J., Zhu, P., Cai, J., Guo, F., y Zhang, Y. (2019). Influence of urban road characteristics on traffic noise. Transportation Research Part D: Transport and Environment, 75, 136-155.

Oyarce, R.E. (2012). Impacto en la salud y calidad de vida por ruido de tráfico vehicular en edificios residenciales de Santiago. Estudio piloto. Unidad de Acústica Ambiental Secretaría Regional Ministerial de Salud Región Metropolitana, Santiago, Chile.

Peña, P.C. (2013). Efectos de la contaminación sonora diurna sobre la población de la ciudad de Barranca (Tesis de licenciatura). Universidad Nacional "José Faustino Sánchez Carrión”, Huacho, Perú.

Peñaloza, I., Flores, A., y Hernández, M. J. (2016). Contaminación acústica en la zona 3 de la ciudad de Querétaro: comparación de los niveles de ruido reales y los apreciados por los habitantes. Entreciencias: diálogos en la sociedad del conocimiento, 4(9), 1-25.

Perea, X., y Marín, E. (2014). Percepción del ruido por parte de habitantes del barrio Gran Limonar de la comuna 17 en la ciudad de Cali (Tesis de pregrado). Universidad del Valle, Cali, Colombia.

Purdy, S.C., y Williams, W. (2002). Development of the Noise at Work Questionnaire to assess perceptions of noise in the workplace. The Journal of Occupational Health and Safety Australia and New Zealand, 18, 77-83.

Quevedo, B.L., y Revolledo, C.K. (2015). Determinación del impacto económico del ruido en el precio de las viviendas de la ciudad de Chiclayo: una aplicación de precios hedónicos (Tesis de pregrado). Universidad Católica Santo Toribio de Mogrovejo, Chiclayo, Perú. 
Ramírez, A., y Domínguez, E.A. (2011). El ruido vehicular urbano: problemática agobiante de los países en vías de desarrollo. Revista Academia Colombiana de Ciencias, 35(137), 509-530.

Ramírez, A., Domínguez, E.A., y Barreto, I. (2011). El ruido vehicular urbano y su relación con medidas de restricción del flujo de automóviles. Revista Academia Colombiana de Ciencias, 35(135), 143-156.

Ramírez, A. (2012). Caracterización y modelación micro y macroscópica del ruido vehicular en la ciudad de Bogotá (Tesis doctoral). Pontificia Universidad Javeriana, Bogotá, Colombia.

Van Renterghem, T. (2018). Towards explaining the positive effect of vegetation on the perception of environmental noise. Urban Forestry \& Urban Greening, 40, 133-144.

Rodríguez, Y., y Baldeón, W. (2018). Evaluación del ruido y el confort acústico en la Biblio teca Agrícola Nacional. Lima, Perú. Medicina y Seguridad en el Trabajo, 64(250), 17-32.

Saavedra, L. (2011). Resultado del estudio subjetivo del ruido y de las mediciones de los niveles de presión sonora en el distrito de Miraflores. Laboratorio de Acústica de la Pontificia Universidad Católica del Perú, Lima, Perú.

Salas, R., y Barbosa, E. (2016). Evaluación del ruido ambiental en el campus de la Universidad Nacional Toribio Rodríguez de Mendoza de Amazonas, Perú. Rev. Indes, 2(1), 88-96.

Santos, E. (2007). Contaminación sonora por ruido vehicular en la Avenida Javier Prado. Industrial Data, 10(1), 11-15.

Solís, I. (2013). Influencia de la contaminación sonora en la salud pública del poblador del Cercado de Lima. PAIDEIA XXI, 3(4), 47-59.

Timaná, M.C. (2017). Nivel de ruido ambiental en el cercado de la ciudad de Piura (Tesis de pregrado). Universidad Nacional de Piura, Piura, Perú.

Vásquez, M. (2017). Influencia de la contaminación sonora en la salud de la población de Cajamarca (Tesis de pregrado). Universidad Privada del Norte, Cajamarca, Perú.

Yagua, W.G. (2016). Evaluación de la contaminación acústica en el centro histórico de Tacna mediante la elaboración de mapas de ruido - 2016 (Tesis de pregrado). Universidad Nacional San Agustín de Arequipa, Arequipa, Perú. 
Yulianto, B., y Saadah, N. (2018). Effect of environmental noise and social environment on the performance of weaving department workers in the textile industry. In The 8th Annual Basic Science International Conference. East Java, Indonesia

Zamorano, B., Velázquez, Y., Peña, F., Ruiz, L., Monreal, O., Parra, V., y Vargas, J. I. (2019). Exposición al ruido por tráfico vehicular y su impacto sobre la calidad del sueño y el rendimiento en habitantes de zonas urbanas. Estudios Demográficos y Urbanos, 34(3), 601-629. 